\title{
Short Communication: Absorption of 2-Hydroxy-4-Methylthiobutanoate in Dairy Cows
}

\author{
H. Lapierre, ${ }^{* 1}$ M. Vázquez-Añón,† D. Parker,† P. Dubreuil,‡ and G. E. Lobley§ \\ *Dairy and Swine Research \& Development Centre, Agriculture and Agri-Food Canada, Sherbrooke, Quebec, Canada, J1M 1 Z3 \\ †Novus International, St. Louis, MO \\ ҒFaculté de médecine vétérinaire, Université de Montréal, St-Hyacinthe, Quebec, Canada, J2S 7C6 \\ §Rowett Research Institute, Aberdeen, UK, AB21 9SB
}

\begin{abstract}
The objective of this study was to measure net portal absorption of 2-hydroxy-4-methylthiobutanoate (HMTBA) in dairy cows. Four multicatheterized lactating cows were used in a cross-over design with 7-d experimental periods. They were fed every other hour a total mixed ration and received, in addition, twice a day, 12.5 or $25 \mathrm{~g} / \mathrm{meal}$ of HMTBA. On the last day of treatment, net portal absorption of HMTBA was numerically greater after the 25 -g compared with the 12.5$\mathrm{g}$ bolus meal of HMTBA, and the amount absorbed relative to the dose ingested was unchanged between treatments averaging $11.2 \pm 4.7 \%$ of the dose. This represents a minimum value of HMTBA availability because it does not take into account any HMTBA metabolized to Met by gut tissues (in sheep this amounted to another $5 \%$ of the dose). A rapid method to estimate net portal absorption based on temporal variations of the peripheral plasma HMTBA concentrations following the HMTBA meal is also presented. Based on the good relationship (concordance correlation coefficient $=0.97$ ) observed between direct measurements and estimations, this simplified approach offers a reasonable approach to assess HMTBA absorption under different feeding situations.
\end{abstract}

Key words: dairy cow, methionine analog, absorption, 2-hydroxy-4-methylthiobutanoate

The release of the NRC (2001) has confirmed that, for many North American diets, Lys and Met are the first-limiting AA in MP for dairy cows under highproducing conditions. Methionine can be supplied directly to the dairy cows via rumen protected Met, but Met supply can also be enhanced through a Met hydroxy analog, DL-2-hydroxy-4-methylthiobutanoate (HMTBA). Although provision of Met through dietary

Received August 22, 2006.

Accepted January 23, 2007.

${ }^{1}$ Corresponding author: lapierreh@agr.gc.ca
HMTBA is fully accepted in nonruminants, a debate still exists over the potential of dietary HMTBA to escape rumen degradation and ultimately provide Met to ruminant tissues. For example, in dairy cows, Koenig et al. $(1999,2002)$ reported ruminal escape of between 41.0 to $50.0 \%$ with concomitant duodenal appearance varying from 16.2 to $44.6 \%$ of bolus doses of HMTBA from 25 to $90 \mathrm{~g}$, the highest escape being observed at the $90-\mathrm{g}$ dose. These results contrast with a recent study in dairy cows, where only $5.3 \%$ of HMTBA intake, mixed in a TMR, passed into the omasum (Noftsger et al., 2005). Measurements of HMTBA flow at different sections of the gut need to be compared with caution due to potential absorption of HMTBA across the rumen or omasal epithelia (McCollum et al., 2000). It should also be remembered that such flows do not necessarily represent absorption, with only $87 \%$ of an abomasal infusion recovered in the portal vein in sheep (Lobley et al., 2006a). Other studies in sheep showed that, on average, $12.5 \%$ of an oral bolus dose of HMTBA was absorbed across the portal-drained viscera, with an additional $5.4 \%$ of the dose estimated to have been used by gut tissues to synthesize Met (Lobley et al., 2006b).

We hypothesized that HMTBA fed as oral bolus doses (as 12.5 and $25 \mathrm{~g}$ per meal) to dairy cows would be absorbed in proportion to the amount ingested. Therefore, our objective was to measure the rate of net portal absorption of ingested HMTBA to estimate the amount absorbed after a bolus meal in lactating dairy cows. A second objective was to further use these measurements to validate the estimation of net portal absorption of HMTBA based on variations of peripheral plasma concentrations of HMTBA, as developed in sheep (Lobley et al., 2006b).

Four multicatheterized cows $(628 \pm 10 \mathrm{~kg}$ of BW, 117 \pm 12 DIM) were used in a crossover design with 7-d experimental periods. The cows were fed twice daily 12.5 or $25.0 \mathrm{~g} / \mathrm{meal}$ (i.e., 25 or $50 \mathrm{~g} / \mathrm{d}$ ) of HMTBA (Alimet, Novus International, St. Louis, MO). At least 3 mo before the start of the study, cows had been implanted with catheters into the portal vein, one hepatic vein, 
and the caudal aorta via a mesenteric artery for blood sampling and in 2 distal mesenteric veins for paraamino hippuric acid (pAH) infusion to determine splanchnic plasma flow (Huntington et al., 1989). On d 7 of each experimental period, a blood sample (time 0 ) was taken at $0730 \mathrm{~h}$ before the meal of HMTBA. At $0800 \mathrm{~h}$, the HMTBA was offered with a small portion of the meal and eaten within 5 min. Then, arterial, portal, and hepatic blood was sampled simultaneously every $20 \mathrm{~min}$ for the next $6 \mathrm{~h}$. During that period, the cows were infused with pAH $(14.4 \mathrm{~g} / \mathrm{h}$ preceded by a priming dose of $2 \mathrm{~g}$ ) into the mesenteric vein beginning at least $40 \mathrm{~min}$ before the first blood sampling. In addition, based on preliminary results, at the end of the 6$\mathrm{h}$ period, sampling from the artery was continued every $30 \mathrm{~min}$ for $2 \mathrm{~h}$ for the 12.5 dose and for $3 \mathrm{~h}$ for the 25.0 $\mathrm{g}$ dose. Throughout the study, based on $95 \%$ of previous ad libitum intake, each cow was fed every $2 \mathrm{~h}$ a fixed amount of TMR, in equal meals, plus $1 \mathrm{~kg} / \mathrm{d}$ of hay, providing 28.6 Mcal of $\mathrm{NE}_{\mathrm{L}}$ and 1,882 $\mathrm{g}$ of MP per d (estimated from NRC, 2001). The diet was balanced to provide sufficient MP and energy but to be deficient in Met (1.83\% of MP). The cows were kept in a tie stall barn and were milked twice a day, at 12-h intervals. The DMI and milk production were recorded daily, and milk samples were collected from the last 4 milkings of each period to analyze milk composition.

Concentrations of plasma HMTBA were determined using the isotopic dilution technique, by GC-MS (Hewlett-Packard, model GC6890-MS5973, Agilent Technologies Inc., Wilmington, DE), with the internal standard DL- $\left[1-{ }^{13} \mathrm{C}\right]$ HMTBA (Wester et al., 2006). Plasma concentrations of pAH were measured with an automatic analyzer (Technicon Autoanalyser II, Technicon Instruments Corporation, NY) as previously described (Huntington, 1984) on fresh samples on the day of sampling. Total absorption of HMTBA was calculated by summation of the absorption of each 20-min interval, calculated as the product of plasma flow times the difference in portal-arterial concentration, from 0 to $360 \mathrm{~min}$.

Absorption of HMTBA in these cows was also estimated using the method developed by Lobley et al. (2006b). Briefly, it is assumed that changes in the HMTBA body pool reflect the differences between inflow (absorption) and outflow. The outflow of HMTBA from the body pool (as conversion to Met or catabolism) is assumed to follow first-order kinetics at a rate equal to $\mathrm{k} \times \mathrm{Q}$, where $\mathrm{k}$ (per min) is the rate constant and $\mathrm{Q}$ is the body pool $(\mu \mathrm{mol})$. The rate constant, $\mathrm{k}$, is estimated as the slope of the decay curve of the natural logarithm of the arterial concentrations of samples collected when net portal absorption of HMTBA had effectively ceased (Figure 1B), that is, 240 min following the meal for the 12.5 dose and 360 min for the 25 dose. The pool Q was
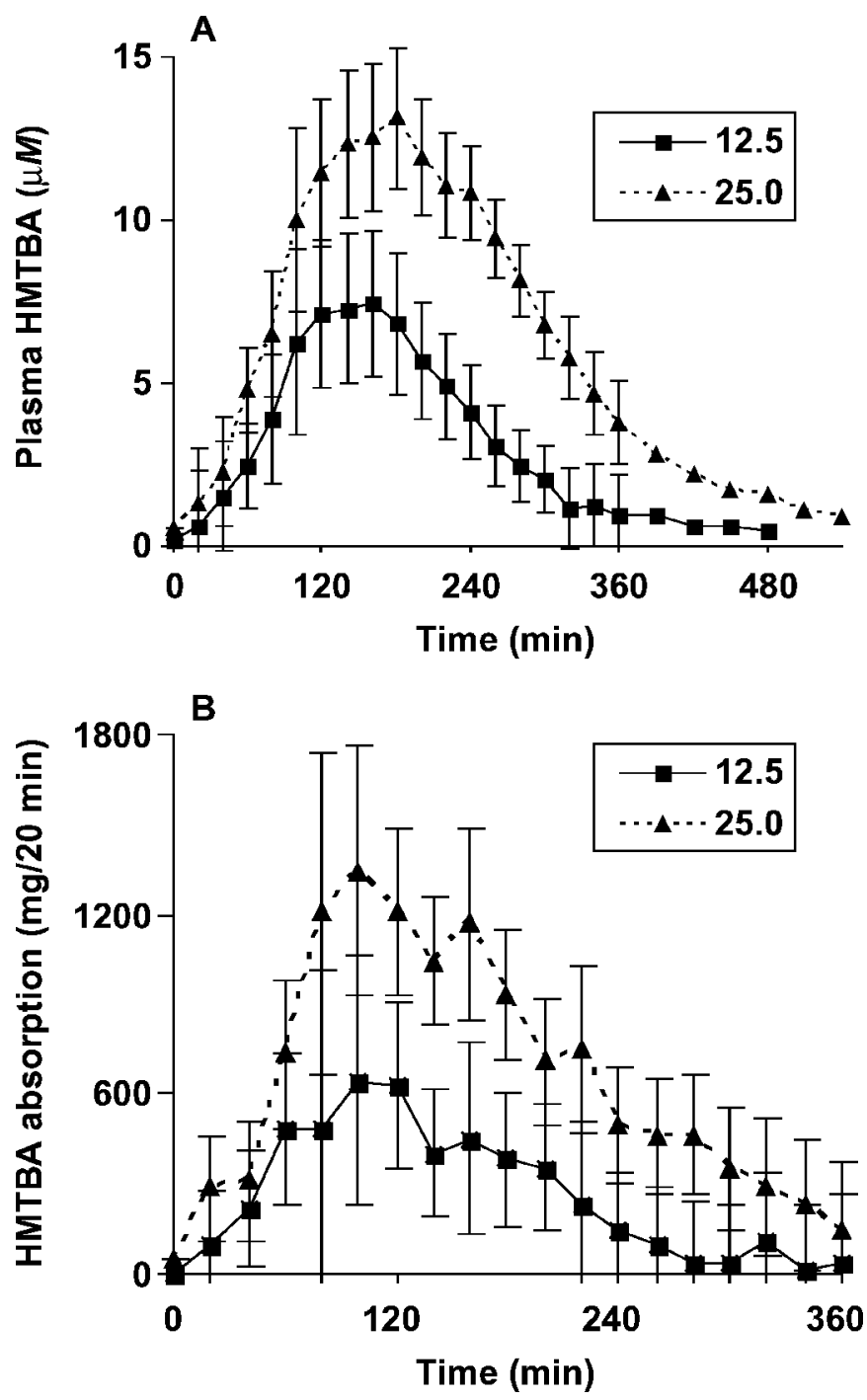

Figure 1. Arterial plasma concentrations (A) and net portal absorptions (B) following a meal of 12.5 or $25.0 \mathrm{~g}$ of 2-hydroxy-4-methylthiobutanoate (HMTBA) in 4 cows. Data are LSM with SEM from 20 to $360 \mathrm{~min}$ (on which statistical analyses were done). For arterial concentrations after $360 \mathrm{~min}$, mathematical means are given due to the impossibility of running statistics on these numbers (see text).

estimated from body water $(\mathrm{BW} \times 0.65)$ multiplied by plasma HMTBA concentrations. Therefore, absorption is estimated as changes in body pool minus outflow. The final estimation was then adjusted by dividing by 0.922 , to account for the first pass liver removal of $7.8 \%$ of the total inflow observed in this study. Data were analyzed according to a crossover design using the MIXED procedure of SAS Institute Inc. (Cary, NC) with treatments and periods as fixed factors and cows as a random factor for the values averaged by cow $\times$ period (splanchnic flux, $\mathrm{k}$ value, and percentage of absorption). Time was added as a fixed factor in repeated measure- 
Table 1. Cumulative net flux (g) of 2-hydroxy-4-methylthiobutanoate (HMTBA) across the splanchnic bed during the $6 \mathrm{~h}$ following a meal of HMTBA $^{1}$

\begin{tabular}{|c|c|c|c|c|}
\hline \multirow[b]{2}{*}{ Tissue bed } & \multicolumn{2}{|c|}{ HMTBA fed (g/meal) } & \multirow[b]{2}{*}{ SEM } & \multirow[b]{2}{*}{$P$-value ${ }^{2}$} \\
\hline & 12.5 & 25.0 & & \\
\hline Portal-drained viscera & 1.24 & 2.51 & 0.77 & 0.22 \\
\hline Liver & -0.41 & -0.89 & 0.27 & 0.23 \\
\hline Splanchnic & 0.83 & 1.60 & 0.50 & 0.22 \\
\hline
\end{tabular}

${ }^{1}$ Least squares means presented with pooled SEM, $n=4$; net portal and hepatic fluxes for HMTBA of 1 cow estimated from her splanchnic flux with hepatic removal of the 3 other cows.

${ }^{2}$ Probability corresponding to the null hypothesis.

ment to assess temporal variations of arterial concentrations and net portal absorption from 20 to 360 (after which the concentrations were below detection limit for 2 cows when fed the lowest dose and portal net absorption measurement has ceased) using the autoregressive heterogeneous covariance structure with the Kenward and Rodger adjustment for the denominator degrees of freedom. One cow did not have a patent portal catheter, and her net portal flux was estimated from the measured splanchnic flux and using a ratio of net portal absorption to splanchnic flux of 1.56, based on the average liver removal observed with the other cows. Treatment differences were considered significant if $P \leq 0.10$ and as a trend for $0.10<P \leq 0.20$. The relationship between the direct measurements of net portal absorption and estimations with variations in peripheral concentrations was evaluated with the concordance correlation coefficient (Lin, 1989).

The dose of HMTBA fed did not affect $(P>0.20)$ DMI $(17.8$ vs. $17.0 \pm 0.76 \mathrm{~kg} / \mathrm{d})$, milk production (30.0 vs. $29.6 \pm 0.91 \mathrm{~kg} / \mathrm{d}$ ), milk protein yield (981 vs. $954 \pm 36.2$ $\mathrm{g} / \mathrm{d}$ ), or milk fat yield (961 vs. $1,033 \pm 53.9 \mathrm{~g} / \mathrm{d}$ ). The temporal changes in arterial plasma concentrations of HMTBA and absorptions measured directly from portal-arterial differences and plasma flow are depicted in Figure 1 (A and B). The average net fluxes of HMTBA across the portal-drained viscera, the liver, and the total splanchnic bed for the 6 -h period following the meal are presented in Table 1 . Following the meal, HMTBA plasma concentrations increased sharply (time effect, $P<0.001)$ and were greater $(P=0.08)$ overall across all sampling times for the larger dose (3.84 vs. $8.15 \pm 1.44 \mu M)$ but with no time $\times$ treatment interaction $(P>0.20)$. Although net portal absorption was only numerically greater $(P=0.22)$ after the 25.0 -g compared with the 12.5-g meal, the proportions of the 2 doses recovered were similar, averaging 11.1 and $11.3 \pm 2.3 \%$ of the dose ingested, for the 12.5- and 25-g dose, respectively. This is similar to the average recovery observed in sheep, $12.5 \%$ of the dose ingested, across a 4 -fold range of HMTBA supply (Lobley et al., 2006b). However, these values of HMTBA availability do not take into account the HMTBA that is metabolized to Met in the portal-drained viscera tissues, a metabolic fate representing $5.4 \%$ of the dose in sheep gut tissue (Lobley et al., 2006b). If similar rates of metabolism occurred in the dairy cow then this would raise true absorption to $16.5 \%$ of the dose. This adjusted value is close to the $16.2 \%$ of the dose ingested that appeared at the duodenum following an intraruminal dose of $25 \mathrm{~g}$ of HMTBA (Koenig et al., 2002) but is less than estimates based on rumen outflow, averaging $45 \%$ for doses ranging between 25 and $90 \mathrm{~g}$ (Koenig et al., 1999, 2002). Because HMTBA can be absorbed from ruminal and omasal epithelium (McCollum et al., 2000), Koenig et al. (2002) had proposed that the difference between rumen outflow and duodenal flow represented ruminalomasal-abomasal absorption, but this would be included in the measurement of net portal absorption used in the current study. The current measurements, however, are larger than those reported for omasal flows (5.3\% of the ingested dose of $20.5 \mathrm{~g}$; Noftsger et al., 2005).

Several factors might explain the difference between the various studies and help resolve the debate concerning the effectiveness of dietary HMTBA to supply Met to ruminant tissues. First, all of these studies were conducted with a relatively small number of cows, and large variations can occur between animals. In the current study, absorption relative to the ingested dose varied between cows from 2.1 to $22.4 \%$ (with a betweenanimal variance of $8.0 \%$ ). Such variation was also observed in sheep, with absorption ranging from 10.6 to $27.9 \%$ of the dose ingested (Lobley et al., 2006b). Cows were consistent, however, in that those that absorbed the least and the most did so for both doses. It is interesting to note that the cow with the lowest absorption rate ate the least (DMI of $15.5 \mathrm{~kg} / \mathrm{d}$ ), whereas the 2 cows eating the most had the highest absorption rates (DMI of $20.0 \mathrm{~kg} / \mathrm{d}$ ). Total intake is known to directly affect rumen outflow (NRC, 2001), and in continuous culture fermenters higher liquid outflow rates increased the amount of HMTBA in the effluent, even though the fractional rate of microbial degradation of HMTBA was unaltered (Vázquez-Añón et al., 2001). Thus, rate of passage and, inversely, retention time in the rumen may affect the amount of HMTBA bypassing the rumen. Most of the HMTBA bypassing the rumen should be absorbed, as shown in sheep where abomasal infusion of HMTBA resulted in recoveries in the portal vein in excess of $85 \%$ (Lobley et al., 2006a). Nonetheless, other factors must also affect HMTBA absorption because the Koenig et al. (2002) and Noftsger et al. (2005) studies reported similar DMI (19.7 and $19.5 \mathrm{~kg} / \mathrm{d}$, re- 
spectively). One such factor might involve feeding pattern. In our study and that of Noftsger et al. (2005), cows were fed 12 times per day, and this might have decreased the rumen outflow compared with feeding once or twice a day and resulted in lower recoveries than observed by Koenig et al. (2002). The way HMTBA is provided to the animal may also be critical. A bolus ingestion (our study) or rumen feeding (Koenig et al., 1999,2002 ) might result in substantial differences compared with incorporation into a TMR (Vázquez-Añón et al., 2001; Noftsger et al., 2005). Finally, the approaches used in these various studies each have different limitations. For example, rumen outflow is not a direct measurement of absorption; omasal and duodenal flows do not account for absorption occurring before HMTBA has reached these sites, whereas net portal absorption does not include any HMTBA transformed into Met by gut tissues.

Because variation in plasma Met concentrations is an invalid method to determine HMTBA availability to the animal (Lobley et al., 2006a,b), another approach, based on variations of HMTBA concentrations in peripheral plasma, has been proposed to indirectly estimate HMTBA absorption (Lobley et al., 2006b). The relationship between the natural logarithm of arterial concentrations and time was linear $(r \geq 0.99)$, and this was used to determine the rate constant, $\mathrm{k}$, for each cow in each period. The half-lives of HMTBA, derived from $\mathrm{k}$, averaged 64 and $77 \pm 5.0 \mathrm{~min}$ for the 12.5 and 25 meals. These values were not affected $(P>0.20)$ by dose level and are similar to those measured in sheep (76 min; Lobley et al., 2006b). Comparison of the measurements of the net portal absorption across the 2 doses for the 3 cows with patent catheters and the estimation of absorption as the difference between the variation in pool size and outflow gave a good agreement, with a concordance correlation coefficient of 0.97 between the measured vs. estimated net portal absorption. Measurement and estimation of the net portal absorption averaged $8.80 \mathrm{vs} .8 .60 \mathrm{mmol} / \mathrm{h}$, respectively, with a ratio of measured/estimated ranging from 0.82 to 1.24. Therefore, as in sheep (Lobley et al., 2006b), this simpler approach could be applied to estimate absorption following bolus ingestion.

In summary, on average $11.2 \%$ of the ingested dose of HMTBA (12.5 and $25 \mathrm{~g}$ ) eaten as a bolus reached the portal circulation in cows fed 12 times per day. This represents a minimum: taking into account HMTBA used by the gut tissues to synthesize Met, estimated in a previous study with sheep, would increase HMTBA availability to $16.5 \%$ of the dose ingested. Estimation of absorption from circulating concentrations offers a simple but reasonable alternative to assess the impact of different factors on HMTBA absorption in dairy cows.

\section{ACKNOWLEDGMENTS}

The authors gratefully thank the staff of the Dairy and Swine Research \& Development Centre for taking care of the animals, M. Léonard and J. Renaud for their dedicated technical support, and S. Méthot for statistical analyses. This work was supported by Novus International and the Matching Investment Initiative of Agriculture and Agri-Food Canada, contribution number 913.

\section{REFERENCES}

Huntington, G. B. 1984. Net absorption of glucose and nitrogenous compounds by lactating Holstein cows. J. Dairy Sci. 67:19191927.

Huntington, G. B., C. K. Reynolds, and B. H. Stroud. 1989. Techniques for measuring blood flow in splanchnic tissues of cattle. J. Dairy Sci. 72:1583-1595.

Koenig, K. M., L. M. Rode, C. D. Knight, and P. R. McCullough. 1999. Ruminal escape, gastrointestinal absorption, and response of serum methionine to supplementation of liquid methionine hydroxy analog in dairy cows. J. Dairy Sci. 82:355-361.

Koenig, K. M., L. M. Rode, C. D. Knight, and M. Vázquez-Añón. 2002. Rumen degradation and availability of various amounts of liquid methionine hydroxy analog in lactating dairy cows. J. Dairy Sci. 85:930-938.

Lin, L. I.-K. 1989. A concordance correlation coefficient to evaluate reproducibility. Biometrics 45:255-268.

Lobley, G. E., T. J. Wester, A. G. Calder, D. S. Parker, J. J. Dibner, and M. Vázquez-Añón. 2006a. Absorption of 2-hydroxy-4-methythiobutyrate (HMTBA) and conversion to methionine in lambs. J. Dairy Sci. 89:1072-1080.

Lobley, G. E., T. J. Wester, G. Holtrop, J. J. Dibner, D. S. Parker, and M. Vázquez-Añón. 2006b. Absorption and digestive tract metabolism of 2-hydroxy-4-methythiobutanoic acid in lambs. J. Dairy Sci. 89:3508-3521.

McCollum, M. Q., M. Vázquez-Añón, J. J. Dibner, and K. E. Webb, Jr. 2000. Absorption of 2-hydroxy-4-(methylthio)butanoic acid by isolated sheep ruminal and omasal epithelia. J. Anim. Sci. 78:1078-1083.

Noftsger, S., N. R. St-Pierre, and J. T. Sylvester. 2005. Determination of rumen degradability and ruminal effects of three sources of methionine in lactating cows. J. Dairy Sci. 88:223-237.

NRC. 2001. Nutrient Requirements of Dairy Cattle. 7th rev. ed. Natl. Acad. Sci., Washington, DC.

Vázquez-Añón, M., T. Cassidy, P. McCullough, and G. A. Varga. 2001. Effects of Alimet on nutrient digestibility, bacterial protein synthesis, and ruminal disappearance during continuous culture. J. Dairy Sci. 84:159-166.

Wester, T. J., M. Vázquez-Añón, J. J. Dibner, D. S. Parker, A. G. Calder, and G. E. Lobley. 2006. Hepatic metabolism of 2-hydroxy4-methythiobutyrate in growing lambs. J. Dairy Sci. 89:10621071. 